\section{Challenges for a sustainable financial foundation for antimicrobial stewardship}

\author{
Jan-Willem H. Dik, Bhanu Sinha \\ Department of Medical Microbiology, \\ University of Groningen, University \\ Medical Center Groningen, the \\ Netherlands
}

\begin{abstract}
Antimicrobial resistance is a worldwide threat and a problem with large clinical and economic impact. Antimicrobial Stewardship Programs are a solution to curb resistance development. A problem of resistance is a separation of actions and consequences, financial and clinical. Such a separation makes it difficult to create support among stakeholders leading to a lack of sense of responsibility. To counteract the resistance development it is important to perform diagnostics and know how to interpret the results. One should see diagnostics, therapy and resistance as one single process. Within this process all involved stakeholders need to work together on a more institutional level. We suggest therefore a solution: combining diagnostics and therapy into one single financial product. Such a product should act as an incentive to perform correct diagnostics. It also makes it easier to cover the costs of an antimicrobial stewardship program, which is often overlooked. Finally, such a product involves all stakeholders in the process and does not lay the costs at one stakeholder and the benefits somewhere else, solving the misbalance that is present nowadays.
\end{abstract}

\section{Introduction}

Antimicrobial resistance (AMR) is a worldwide threat and problem. Loss of effectiveness of antimicrobial therapy leads to untreatable infections and is causing already substantial loss of life., ${ }^{1,2}$ This is expected to grow even more in the near future. The economic impact is consequently also vast. ${ }^{2,3}$ A plan of action is thus needed and this should focus on all aspects of infection management as well as on public awareness and involvement of pharmaceutical industries, but also veterinary and agriculture industries; it requires thus a worldwide multi-sector response. ${ }^{2,4}$ With regards to hospitals, Antimicrobial Stewardship Programs (ASPs) are hailed as one the solutions to tackle the development of AMR. ${ }^{5,6}$ These programs focus on improvement of therapy by means of a set of diverse interventions. An important aspect is correct diagnostics, without diagnostics physicians are treating patients in fact blind. However, implementation of these programs in healthcare institutions is often a difficult and long process. ${ }^{7}$ One of the reasons for this difficult implementation might lie in the fact that provided funds are insufficient. We provide here an overview of the economic aspects with regards to ASPs and possible solutions from a financial point of view.

\section{The misbalance of actions and consequences}

One of the main problems of antimicrobial resistance is the fact that actions and consequences are separated from each other. Not just in time, but also in stakeholders. This makes it more difficult to implement actions and create support. In hospitals, clinical and financial effects from suboptimally antimicrobial use and the consequent AMR often fall upon other departments or healthcare providers than the initiators of these problems (i.e. the prescribers). This creates a lack of sense of responsibility for these problems and is translated into the fact that diagnostics are often not performed according to guidelines and also antimicrobial therapy is often suboptimally prescribed. The problems of these actions occur often on the long(er) term, are not directly visible, and others most likely bear the consequences. These consequences include patients with difficult to treat infections caused by resistant microorganisms. If such an infection occurs, the respective patient often already left the ward where therapy was given initially and in some cases even left the hospital. It therefore might well be another hospital that has to deal with this infected/colonized patient and all the extra measures and costs such as isolation and more expensive therapy. It also makes difficult to convince healthcare providers (or the prescribing department, depending on the cost structure) to spend money on the correct diagnostics and interpretation of these diagnostics (e.g. via an ASP), if it means that the benefits of those expenditures lie with other parties. It is therefore imperative to look at antimicrobial use in a more process-based manner, taking into account all stakeholders within the process. Firstly within one healthcare center, however it is also important to collaborate with neighboring healthcare centers and define a healthcare region. ${ }^{8}$ Preferably, all costs and benefits are shared among all stakeholders to remove the misbalance and create a more sustainable funding of both diagnostics and stewardship measures. It is the task of microbiologists,
Correspondence: Bhanu Sinha, University Medical Center Groningen, Hanzeplein 1, $9713 \mathrm{GZ}$, Groningen, the Netherlands.

E-mail: b.sinha@umcg.nl

Key words: Antimicrobial Stewardship, Antimicrobial Resistance, Health Economics.

Contributions: the authors contributed equally.

Conflict of interest: BS has worked on projects in cooperation with Pathogenica and Life Technologies. BS has ongoing collaborations in joint projects with Copand Italia, IDMCore (both EU Horizon 2020 Cofund Pronkjewail), and project partners, e.g. AGILeBiotics (EU Interreg health-i-care).

Received for publication: 28 August 2016. Revision received: 28 December 2016.

Accepted for publication: 29 December 2016.

This work is licensed under a Creative Commons Attribution-NonCommercial 4.0 International License (CC BY-NC 4.0).

CC Copyright J.-W. H. Dik and B. Sinha, 2017 Licensee PAGEPress, Italy

Infectious Disease Reports 2017; 9:6851 doi:10.4081/idr.2017.6851

infectious disease specialists and pharmacists, to work together to achieve these goals crossing borders between treating specialties and between neighboring hospitals and setting aside possible conflicting issues. Only then, is it possible to create a sustainable financial solution for the prevention and management of antimicrobial resistance.

\section{The macroeconomic point-of-view}

In May 2016 the final report of the Review on Antimicrobial Resistance, led by Jim O'Neill, was published. ${ }^{2}$ This is one of the most important reports of the last couple of years, mainly because it addressed the problem from a worldwide, macroeconomic point-of-view. Often, the bigger picture is lost when focusing too much on single patients or institutions. Especially with a worldwide problem such as AMR and with such large economic consequences, it is important to look at the bigger picture. The report once more confirmed the dire situation that the world faces with regards to AMR. The financial impact will be huge, with worldwide costs adding up to billions per year, for a large part due to the loss of workforce of people infected with resistant and untreatable pathogens. Investments to counteract AMR and subsequent problems are expected to be dwarfed by the costs of doing nothing. ${ }^{2}$

One of the main points addressed in the 
report, is the diagnostics, and more specifically the lack of appropriate and timely diagnostics when antimicrobial therapy is started. Before start of therapy, microbiological diagnostics are normally required and asked for in almost all guidelines. However, in many cases, these diagnostics are not performed or not performed on time making them less effective. ${ }^{9-11}$ However, when cultures of biological samples are performed, results can take several days until they become available. There is thus substantial room for improvement in this area. For now, it is important that microbiological diagnostics are performed before start of therapy. For the near future more steps are required to achieve the ideal situation of available rapid diagnostics to minimize the use of broad-spectrum empiric therapy. The AMR Review calls upon the government of the richest countries to achieve these goals in $2020 .^{2}$ It provides some steps to stimulate new rapid diagnostics and how to finance this, but not to improve current use of diagnostics. This is equally important in our view, having diagnostics available is one thing, but prescribers should also use them correctly and interpret them correctly. ${ }^{6}$

\section{Working together towards a solution}

Not only are financial costs and benefits of antimicrobial resistance and antimicrobial misuse divided from each other, but also those of diagnostics and therapy (and thus also of an ASP). This can lead to prescribers (or departments) making the decision not to order diagnostics, because the result will be available when the patient is most likely already transferred to another department. The benefits of these diagnostics but also the consequences of not doing diagnostics often fall therefore not on the prescribers but on others. The question will therefore be how to make sure that process and result is supported by correct (financial) incentives.

It is thus important to view diagnostics, therapy and AMR development as one single decisional process. Within this process all involved stakeholders need to work together on an institutional level. Subsequently, these institutions should work together on a regional level and finally countries should work together on a worldwide level. Nowadays, people can travel within one day across the globe, and carry with them their microorganisms, making spread of AMR far easier than before. It is vital that this high interconnectivity that exits is not forgotten in tackling AMR. ${ }^{8}$

Ideally costs and benefits are looked at from a process-based point of view, including all stakeholders. This is however not realistic to implement in most countries. We suggest therefore an easier to implement solution: combining diagnostics and therapy into one single product. Although this does not completely fix the abovementioned issues, it is a realistic and achievable first step. It would assure that diagnostics are not forgotten or lost due to unfounded budget costs. The costs of these products should fall on the institution and not just on the prescribing department. The internal and national cost structure for healthcare is of influence here. Countries with a diagnosis-based payment system [e.g. with diagnosis related groups (DRGs)] can implement such a single product relatively easy. A combination of therapy and diagnostics partly solves the raised issue of divided cost and benefits, thereby removing the financial incentive not to order diagnostics. Furthermore, if such a combined product is part of a diagnosis, it is also easier for governments to invest in, and subsidize more costly rapid diagnostics (one of the key aspects of the O'Neill report) without the risk of hospitals performing too much diagnostics because they are subsidized. In the end, this proposed package of antimicrobial therapy and diagnostics should improve the possibilities to optimize antimicrobial therapy, reduce unnecessary broad spectrum antibiotics, reduce toxicity and catheterrelated infections and finally reduce AMR development because there are more complete diagnostic results available at an earlier time point. The benefits of all these positive effects not only fall upon the institution that treats the patients of on the whole region in which a patient moves and eventually the whole nation and world.

\section{The role of Antimicrobial Stewardship Programs}

It is worthwhile to notice, that only performing diagnostics is not enough, the interpretation and the logistics are equally important. This means that rapid diagnostics only work, if the laboratory can communicate the results effectively to the responsible physician. Interpretation of diagnostic results and advice on therapy are perfect examples of tasks that can be performed under the umbrella of ASP. However, this brings with it additional costs that should also be paid by someone. Because of the importance of an ASP from a quality point-of-view and the fact that there seem to be some highly effective interventions that they can facilitate, we would suggest to accommodate these costs in the package price of therapy and diagnostics. For the Netherlands for example, an ASP is mandatory for all hospitals. Such an obligation also asks for a way of financing. The AMR Review suggests that govern- ments should be actively involved in financing actions towards preventing AMR, because the costs of doing nothing ultimately also falls on society and thus on the government. ${ }^{2}$ A package price, funded or subsidized by governments (depending on the cost structure present in a country) is in line with this viewpoint. We feel that a consequence of such a package price should be that some kind of control is implemented nationally to ensure that hospitals indeed perform diagnostics and have an active ASP.

\section{Conclusions}

The process of AMR development is a complex one, with many different stakeholders involved. It is not always clear what the exact role and effect is of each of these actors. It is however clear that there is a misbalance in actions and consequences on a clinical level, but as importantly also on a financial level. This misbalance can facilitate the misuse of antimicrobials including the performance of diagnostics. We would therefore suggest a new cost structure to reimburse diagnostics and to dissolve the misbalance and create a financial incentive to perform better quality of care regarding antimicrobial use. This should ultimately positively affect the development of AMR Because of the wide impact of AMR, especially on an economical level, national government should act upon this and contribute in the finances of new techniques and improved quality of care. The introduction of a package price for antimicrobial therapy and diagnostics is a solution that can answer these issues and should therefore be investigated further.

\section{References}

1. Laxminarayan R, Duse A, Wattal C, et al. Antibiotic resistance-the need for global solutions. Lancet Infect Dis 2013;13:1057-98.

2. Review on Antimicrobial Resistance. Tackling drug-resistant infections globally: final report and recommendations. London, UK. 2016. Available from: https://amr-review.org/sites/ default/files/160518_Final\%20paper_w ith $\% 20$ cover.pdf

3. Smith R, Coast J. The true cost of antimicrobial resistance. $\mathrm{Br}$ Med J 2013;346:f1493.

4. Årdal C, Outterson K, Hoffman SJ, et al. International cooperation to improve access to and sustain effectiveness of 
antimicrobials. Lancet 2016;387:296307.

5. Davey P, Brown E, Charani E, et al. Interventions to improve antibiotic prescribing practices for hospital inpatients. Cochrane Database Syst Rev 2013:4;CD003543.

6. Dik JH, Poelman R, Friedrich AW, et al. An integrated stewardship model: antimicrobial, infection prevention and diagnostic (AID). Future Microbiol 2016:93-102.

7. van Limburg M, Sinha B, Lo-Ten-Foe
JR, van Gemert-Pijnen JEWC. Evaluation of early implementations of antibiotic stewardship program initiatives in nine Dutch hospitals. Antimicrob Resistance Infect Control 2014;3:133.

8. Ciccolini M, Donker T, Köck RR, et al. Infection prevention in a connected world: The case for a regional approach. Int J Med Microbiol 2013;303:380-7.

9. Collini P, Beadsworth M, Anson J, et al. Community-acquired pneumonia: doctors do not follow national guidelines. Postgrad Med J 2007;83:552-5.
10. Reissig A, Mempel C, Schumacher U, et al. Microbiological diagnosis and antibiotic therapy in patients with community-acquired pneumonia and acute COPD exacerbation in daily clinical practice: comparison to current guidelines. Lung. 2013;191:239-46.

11. Chia D, Yavari Y, Kirsanov E, et al. Adherence to standard of care in the diagnosis and treatment of suspected bacterial meningitis. Am J Med Qual 2015;30:539-42. 